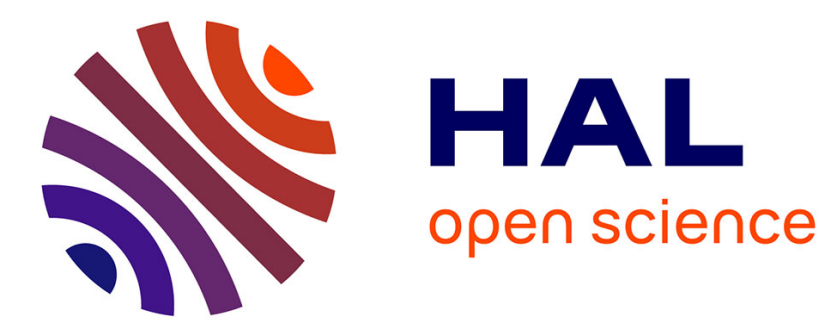

\title{
Grammaire fractale et sémantique transcatégorielle: entre syntaxe et lexique \\ Stéphane Robert
}

\section{To cite this version:}

Stéphane Robert. Grammaire fractale et sémantique transcatégorielle: entre syntaxe et lexique. Langage, 1999, 136, pp.106-123. hal-00076721

\section{HAL Id: hal-00076721 \\ https://hal.science/hal-00076721}

Submitted on 26 May 2006

HAL is a multi-disciplinary open access archive for the deposit and dissemination of scientific research documents, whether they are published or not. The documents may come from teaching and research institutions in France or abroad, or from public or private research centers.
L'archive ouverte pluridisciplinaire HAL, est destinée au dépôt et à la diffusion de documents scientifiques de niveau recherche, publiés ou non, émanant des établissements d'enseignement et de recherche français ou étrangers, des laboratoires publics ou privés. 
Robert, Stéphane, 1999, “Grammaire fractale et sémantique transcatégorielle: entre syntaxe et lexique ", Langages 136 (décembre), numéro consacré à la "Sémantique lexicale et grammaticale " coordonné par Y. Mathieu, 106-123.

Stéphane ROBERT, CNRS-LLACAN

\author{
Grammaire fractale et sémantique transcatégorielle: entre syntaxe et lexique \\ (version non corrigée)
}

Résumé:

The nature of grammatical semantics and its relashionships to lexical semantics are approached here through the problem of synchronic grammaticalization. Synchronic use of morphems in different syntactic categories is then analyzed in terms of fractals and topology, illustrated by examples from Wolof. Thus the functional model of fractal grammar, combining 'scale invariance' and 'scale properties', accounts for the semantic unity and functional flexibility of the transcategorial morphems through a dynamic linking of semantic and syntactic variations.

\title{
Introduction
}

L'étude de la grammaticalisation constitue un champ d'investigation particulièrement intéressant pour essayer de définir la nature du sémantisme grammatical puisqu'elle permet d'observer, sous la forme d'un passage, un lien motivé entre sémantique lexicale et sémantique grammaticale. La plupart du temps, les phénomènes de grammaticalisation sont abordés dans leur aspect diachronique et, à ce titre, certaines analyses posent qu'au cours de l'évolution, il y a une forme de déperdition du sens, la grammaticalisation se caractérisant notamment, selon cette conception, par une forme de désémantisation (Meillet 1912), une sorte de "décoloration" ('bleaching') du signifié (Givón 1975).

Cependant, dans la plupart des langues il existe également des cas de grammaticalisation synchronique sous la forme de morphèmes qui fonctionnent conjointement dans plusieurs catégories grammaticales. Il peut s'agir de termes du lexique qui présentent des emplois grammaticalisés (comme par exemple des noms désignant les parties du corps qui sont utilisés comme relateurs ou comme subordonnants) ou de morphèmes grammaticaux polyfonctionnels qui sont utilisés conjointement dans différentes catégories grammaticales. Dans la mesure où il n'y a pas abandon d'une structuration première au profit d'une autre mais fonctionnement conjoint des différentes structures, on préfèrera ici le terme de "transcatégoriel" pour désigner le fonctionnement synchronique de ces éléments dans différentes catégories syntaxiques.

Ces morphèmes transcatégoriels sont évidemment plus nombreux dans les langues à morphologie faible où l'appartenance catégorielle n'est pas marquée morphologiquement, comme par exemple le chinois (voir notamment Hagège 1975). Mais ils existent d'une manière ou d'une autre dans toutes les langues, dans des proportions variables selon la structure de celles-ci. Ainsi par exemple, même dans des langues flexionnelles comme le français ou l'anglais, on trouve des termes qui fonctionnent avec une portée syntaxique (et un sémantisme) variable, tels quebien (nom, adverbe, connecteur propositionnel), maintenant et 
Robert, Stéphane, 1999, "Grammaire fractale et sémantique transcatégorielle: entre syntaxe et lexique ", Langages 136 (décembre), numéro consacré à la "Sémantique lexicale et grammaticale" coordonné par Y. Mathieu, 106-123.

toujours, tantôt adverbes de temps (déterminant un verbe), tantôt connecteurs argumentatifs (reliant des propositions), ou encore d'ailleurs ou any way/anyway. Le fait que ce glissement de catégorie se produise en synchronie place alors la transcatégorialité au niveau du système de la langue.

Ces morphèmes transcatégoriels permettent de saisir "in vivo" l'apparentement entre sémantique lexicale et sémantique grammaticale et posent le problème de la construction du sens dans sa difficulté maximale: comment définir à la fois l'unité sémantique du terme et la spécificité indéniable de chacun des emplois? Il faut en effet pouvoir expliquer ce qui change dans la signification du terme à travers ses différents emplois, ce qui se perd et ce qui perdure, mais aussi ce qui se crée d'une catégorie à l'autre et, plus généralement, ce qui, dans le système, permet ces variations. Pour rendre compte des emplois aussi différenciés d'un même terme, où la variation est à la fois sémantique et fonctionnelle, on a besoin d'un modèle de construction de la signification à la fois unificateur et malléable.

On proposera ici un modèle, celui de la "grammaire fractale" ${ }^{1}$, qui tente de rendre compte de cette malléabilité sémantique et syntaxique pour expliquer à la fois l'apparentement et les différences entre les divers emplois de ces morphèmes transcatégoriels. Ce modèle fait intervenir les notions de forme schématique et de portée syntaxique, reformulées en termes de variation d'échelle, d'invariance d'échelle et de propriétés d'échelle. Cette étude qui s'appuie principalement sur deux marqueurs du wolof sera donc l'occasion d'observer la construction d'une sémantique grammaticale à travers le fonctionnement de différents morphèmes transcatégoriels et de définir ce qui relie et ce qui distingue sémantique lexicale et sémantique grammaticale.

\section{VARIATION DE PORTEE ET VARIATION DE SENS}

La flexibilité syntaxique est une caractéristique de ces morphèmes transcatégoriels. Dans leurs différents emplois, en effet, la portée syntaxique de ces termes varie et, avec elle, leur incidence sémantique. On peut décrire ce fonctionnement transcatégoriel en termes de "variation d'échelle". Par "échelle syntaxique" j'entends ici le niveau où opère le terme dans la hiérarchie des relations syntaxiques: ce niveau est défini par la portée syntaxique du terme ('syntactic scope') et entraîne une variation de sa portée sémantique. Par rapport au terme de "portée", la notion d'échelle syntaxique permet d'introduire l'idée d'une géométrie variable du domaine d'application du sémantisme du terme. Dans le cas des morphèmes transcatégoriels, l'échelle syntaxique varie pour chaque type d'emploi, ce qui suppose une dynamique de passage d'une échelle syntaxique à une autre. Or cette variation d'échelle joue un rôle dans la variation du sens du terme.

Pour éclairer la notion de variation d'échelle, je présenterai d'abord le cas d'un suffixe spatial du wolof qui apparaît à la fois dans le système nominal, dans le système verbal et dans la formation de morphèmes subordonnants. Ce morphème est ainsi utilisé pour la localisation

\footnotetext{
${ }^{1}$ La primeur de cette expression revient, je crois, à l'enseignement oral de David Cohen à qui j'exprime ici ma dette indirecte. Il me semble néanmoins qu'il ne lui attribue pas le même sens et l'emploi que j'en fais relève de ma seule responsabilité. En outre, j'ai développé ce modèle dans le cadre des travaux sur la transcatégorialité menés au sein de l'équipe LLACAN qui seront présentés dans un volume collectif, Polysémie, recatégorisation et échelles syntaxiques, à paraître chez Peeters. Enfin, je tiens à remercier également Alexandre François pour la relecture critique dont a bénéficié cet article.
} 
Robert, Stéphane, 1999, "Grammaire fractale et sémantique transcatégorielle: entre syntaxe et lexique", Langages 136 (décembre), numéro consacré à la "Sémantique lexicale et grammaticale " coordonné par Y. Mathieu, 106-123.

d'un objet dans l'espace, mais aussi pour l'expression de relations syntaxiques et pour la prédication. J'aborderai ensuite le passage du lexique à la grammaire avec un autre morphème du wolof (2).

\subsection{Espace déictique et espace syntaxique (l'exemple du wolof -u)}

Le wolof (langue africaine du groupe ouest-atlantique, parlée principalement au Sénégal) possède un suffixe -u dont les emplois traversent l'ensemble du système de la langue puisqu'il est employé à la fois comme connecteur "génitif", dans la formation des pronoms relatifs et interrogatifs, dans celle des conjonctions à valeur hypothétique et temporelle, mais aussi dans la formation de conjugaisons (négatives) et de suffixes verbaux (négatif et passif-réfléchi). Ce morphème fait partie d'un paradigme de trois suffixes spatiaux $-\mathrm{i},-\mathrm{a},-\mathrm{u}$. Pour une analyse détaillée de emplois de ce triplet d'indices spatiaux du wolof, on pourra se reporter à Robert (1998). On se contentera ici de résumer le cas de -u qui présente les emplois les plus divers. Il est en effet employé, seul ou en combinaison, pour former les sept éléments suivants:

\begin{tabular}{|l|}
\hline (1) connectif (complément de nom) \\
(2) relatif indéfini \\
(3) pronom interrogatif \\
(4) subordonnant à valeur future \\
(5) subordonnant à valeur $-u$ : $\quad \begin{array}{l}\text { hypothétique } \\
\text { (6) négation } \\
\text { (7) suffixe passif- réfléchi }\end{array}$ \\
\hline
\end{tabular}

Le morphème -u apparaît ainsi "à nu", c'est-à-dire sans autre morphème grammatical, lorsqu'il est suffixé à un nom; il fonctionne alors comme connectif:

(1) doomu buur

fruit-u roi

(un) fils/fille de roi

Suffixé à un classificateur ${ }^{2}$ (ici la consonne b-), il prend la valeur de relatif indéfini:

(2a) dama bëgg piis bu xonq

EmphVb.1sg vouloir pièce-de-tissu class.+u (Aor 3.sg=Ø) être-rouge

je veux un tissu qui soit rouge

(2b) xam na xale bu dem Tugël

connaître Pft.3sg enfant class.+u aller (Aor 3.sg) France

il connaît un enfant qui est allé en France

\footnotetext{
${ }^{2}$ Le wolof a huit "genres" (huit classes du singulier) auquels correspondent deux pluriels (deux classes pour le pluriel). Le morphème de classe se présente sous la forme d'une consonne $C$ - qui n'a pas d'existence autonome: $k-, b-, g-, j-, w-, m-, s-, l-$ pour le singulier, et $y$ - et $\tilde{n}$ - pour le pluriel. Le rôle des classes concerne essentiellement la détermination nominale. Ainsi, le défini, qui est postposé au nom, est construit à l'aide du morphème de classe (la consonne) auquel est suffixé un indice de détermination par rapport à l'espace du locuteur.
} 
Robert, Stéphane, 1999, "Grammaire fractale et sémantique transcatégorielle: entre syntaxe et lexique ", Langages 136 (décembre), numéro consacré à la "Sémantique lexicale et grammaticale " coordonné par Y. Mathieu, 106-123.

On comparera le relatif indéfini bu à l'article défini bi (cf note 2), dans xale bi "l'enfant à proximité", et au relatif défini bi dans l'exemple suivant:

(2c) xam na xale bi dem Tugël connaître Pft.3sg enfant class.+i aller (Aor.3sg) France

il connaît l'enfant qui est allé en France (relative définie)

Suffixé à un classificateur (cf note 2), ici k-, à l'initiale d'énoncé, -u permet de former un pronom interrogatif (ici ku):

(3) Ø Ku jël saabu bi ? Ø qui (Aor.3sg) prendre savon le $\emptyset$ Qui a pris le savon ?

Suffixé au morphème subordonnant b-, il sert à former un subordonnant à valeur future:

(4) Bu demee dëkk ba, na jënd ma piisu mailus

quand+indét. (Aor.3sg) aller+antér ville la+éloignt, Oblig.3sg acheter me pièce+connectif tissu-bleu

Quand il ira à la ville, qu'il m'achète une pièce de tissu bleu

Suffixé au morphème subordonnant b- ou s-, il sert à former un subordonnant à valeur hypothétique:

(5) Dinaa ko ko wax bu / su ñówee

Futur+1sg le lui dire si (Aor) venir+antériorité

Je le lui dirai s'il vient

Enfin, lorsqu'il a une incidence sur le verbe, -u constitue le morphème de négation du wolof qui apparaît dans différentes structures négatives, conjugaisons (cf 6a) ou affixe négatif $(6 \mathrm{~b})^{3}$ :

(6a) Feccuma

danser+Nég.acc.1sg

(conjugaison Négatif accompli)

Je ne danse pas (actuellement)

(6b) Maa naanul

EmphS.1sg boire+nég

(suffixe de négation -ul)

C'est moi qui n'ai pas bu

Je propose également de voir dans le suffixe -u de passif-réfléchi, ce même morphème spatial:
gaañ blesser
gaañu
"se blesser, être blessé"
wat raser
watu "se raser, être rasé"
yar éduquer, élever
yaru "être (bien) élevé, être poli"

\footnotetext{
${ }^{3}$ La négation en wolof constitue un système complexe et hétérogène avec, d'un côté, des affixes négatifs qui se combinent à des conjugaisons affirmatives (6b) et, de l'autre, de véritables conjugaisons négatives (6a). L"élément commun à ces différents monèmes négatifs et qui porte la valeur de négation est ce morphème $-u$. Pour une présentation générale du système, voir Robert 1990a ou 1991.
} 
Robert, Stéphane, 1999, "Grammaire fractale et sémantique transcatégorielle: entre syntaxe et lexique ", Langages 136 (décembre), numéro consacré à la "Sémantique lexicale et grammaticale " coordonné par Y. Mathieu, 106-123.

A-t-on bien affaire ici à une seule unité? Et quelle est alors la valeur sémantique qui fonde l'unité de ce morphème, aux travers de ses différents emplois? L'apparentement entre les différents emplois est clair si l'on part d'une valeur centrale liée à l'absence de localisation spatiale, comme nous allons le montrer. Cet apparentement est, de plus, corroboré par le fait que -u fait partie d'un système d'indices à valeur spatiale $(-i,-a,-u)$ et que, dans la plupart de ses emplois, il commute de manière significative avec ces autres morphèmes spatiaux (cf 1.2 et Robert 1998, 1991: 276-7). En outre, en ce qui concerne plus spécifiquement -u, on retrouve dans d'autres langues apparentées au wolof, un morphème qui présente une diversité d'emploi comparable. Ainsi, en peul, il existe un morphème A, défini par J.C. Hilaire (1995: 33-192) comme marqueur de non délimitation et d'alocalisation temporelle, qui apparaît notamment dans des formes verbales à valeur d'éventuel, d'hypothétique, de passif, de négatif ainsi que dans la formation des relatives indéfinies.

\subsection{La portée syntaxique: premier facteur de variation}

Le morphème -u rentre, en effet, dans un triplet d'indices de détermination par rapport à l'espace du locuteur (Sauvageot: 77-70) qui correspondent aux trois cas suivants:

-i proximité par rapport au locuteur

-a éloignement par rapport au locuteur

-u indétermination par rapport à l'espace du locuteur

Ces indices spatiaux sont des déictiques dont les emplois traversent l'ensemble du système de la langue. Ils servent notamment à former l'article défini avec une valeur spatiale claire. Ainsi, suffixés à la consonne de classe, -i sert à former le défini proche et -a le défini éloigné:
(8) xale bi
l'enfant (à proximité du locuteur)
xale ba
xale yi / ya
l'enfant (éloigné du locuteur)
les enfants proches / éloignés

En wolof, la détermination nominale comporte donc nécessairement une spécification concernant la position dans l'espace de l'objet désigné, par rapport au locuteur. De manière remarquable, le suffixe -u d'indétermination par rapport à l'espace du locuteur ne sert pas à construire l'indéfini et le syntagme /nom Classificateur $+u /$ seul est impossible. En effet, ${ }^{*} x a l e$ bu ne forme pas un syntagme complet, on attend une détermination supplémentaire: il faut que le nom soit complété par un autre syntagme et le classificateur muni de -u fonctionne alors comme pronom relatif indéfini ${ }^{4}$ :

$$
\begin{array}{ll}
* \text { xale bu } & \rightarrow \quad \text { xale bu dem Tugël (cf 2) } \\
& \text { un enfant qui est allé en France }
\end{array}
$$

Dans la détermination nominale, l'indétermination par rapport à l'espace du locuteur construit donc deux spécifications: (1) elle sert à exprimer le caractère indéfini du nom, (2) elle construit une dépendance syntaxique entre le nom déterminé et le syntagme qui suit. Cette caractéristique syntaxique est cruciale et s'explique par le sémantisme du terme.

En effet, dans les constructions linguistiques, tout terme entrant dans une relation doit être situé (avoir un "site"), c'est-à-dire stabilisé dans un schéma relationnel, par rapport à un autre

\footnotetext{
${ }^{4}$ Pour plus de détails sur le système complexe des relatives en wolof, voir Robert (1998).
} 
Robert, Stéphane, 1999, "Grammaire fractale et sémantique transcatégorielle: entre syntaxe et lexique ", Langages 136 (décembre), numéro consacré à la "Sémantique lexicale et grammaticale" coordonné par Y. Mathieu, 106-123.

terme (Culioli, 1990: 119). Ceci vaut quelle que soit la nature du terme: syntagme nominal ou énoncé. De ce fait, lorsqu'une relation prédicative n'est pas située par rapport à l'espace-temps de l'énonciation, l'énoncé est incomplet (Culioli [1978] 1990: 35-155) et en attente de repérage situationnel, ce qui se traduit syntaxiquement par une dépendance situationnelle (Robert 1996): c'est alors un élément extérieur qui donnera son site à la relation prédicative.

Cette caractéristique générale du fonctionnement des langues permet de comprendre les différents emplois de -u.. Ce morphème indique précisément une absence de localisation dans l'espace-temps de l'énonciateur. Cette absence de site entraîne alors une dépendance par rapport à un autre terme qui lui donnera son site: ce deuxième terme peut-être un nom (d'où l'emploi comme connectif) ou une relation prédicative (d'où ses divers emplois comme subordonnant).

-u indétermination spatiale: absence de localisation dans l'espace-temps du locuteur $\rightarrow$ recherche d'un site $\rightarrow$ dépendance syntaxique

Lorsque -u introduit une relation prédicative, on a deux configurations possibles qui produisent des valeurs syntaxiques et sémantiques spécifiques (Robert 1998). Si la relation prédicative ne s'articule à aucune autre proposition, elle sera alors en attente absolue d'ancrage situationnel et prendra une valeur d'interrogation ${ }^{5}$ (recours à autrui pour assigner un ancrage situationnel); si la relation prédicative s'articule à une autre proposition, on a ce que j'ai appelé une anaphore situationnelle (Robert 1996): la relation prédicative en question est rattachée à la situation d'une autre proposition. A partir de cette analyse, les différents emplois du morphème -u peuvent s'expliquer par la portée syntaxique variable du suffixe.

- Ainsi, lorsque l'indétermination porte sur un argument, on a un fonctionnement comme connectif (exemple 1) ou comme pronom relatif indéfini ${ }^{6}$ (exemple 2), suivant la nature de ce qui suit, nom ou proposition; ou encore comme interrogatif (exemple 3), si le suffixe ne s'appuie sur aucun argument appartenant à une proposition qui possède un site.

- Lorsque l'indétermination porte sur le paramètre spatio-temporel d'un énoncé, comme lorsque -u est suffixé au subordonnant temporel b-(ou s-), on a un fonctionnement comme subordonnant à valeur d'éventuel (exemple 4 "quand il ira à la ville, qu'il m'achète une pièce de tissu bleu") ou d'hypothétique (exemple 5 "je le lui dirai s'il vient") ${ }^{7}$. Avec -u, la proposition en question est posée dans une situation qui est indéterminée par rapport à l'espace du locuteur et donc située sur un autre plan que celui de l'énonciation, à savoir celui de l'éventualité ou de l'hypothèse.

- Enfin, lorsque l'indétermination spatiale porte sur le verbe en fonction prédicative (cf place de -u), le procès est déterminé comme non localisé dans la situation d'énoncation, il n'est donc pas vrai au moment où je parle (exemple 6). Lorsque -u est suffixé au lexème verbal

\footnotetext{
${ }^{5} \mathrm{Ou}$, le cas échant, d'injonction; ce n'est pas le cas pour $-u$ mais pour une conjugaison, l'Aoriste, qui a cette valeur particulière de localisation dans une situation qui doit être spécifiée par ailleurs (Robert 1996).

${ }^{6}$ On notera que s'il n'y a pas d'antécédent, le pronom prend une valeur de générique comme dans : $\varnothing$ Ku yàgg dox, yàgg gis /qui (Aor.) durer marcher, (Aor) durer voir/: "Celui qui (= tout homme qui) marche longtemps, voit beaucoup de choses" (Le voyage donne de l'expérience). La proposition relative est alors vraie quelle que soit la situation.

${ }^{7}$ Dans ces emplois $-u$ commute avec $-i$ et $-a$ dans $b i$ et $b a$ qui servent respectivement à exprimer une temporelle proche/éloignée du moment de l'énonciation ( $b i$ "quand (il y a peu de temps)", $b a$ "quand (moment éloigné de maintenant)").
} 
Robert, Stéphane, 1999, "Grammaire fractale et sémantique transcatégorielle: entre syntaxe et lexique ", Langages 136 (décembre), numéro consacré à la "Sémantique lexicale et grammaticale " coordonné par Y. Mathieu, 106-123.

(exemple 7), l'indétermination spatio-temporelle ne porte plus sur la prédication ou sur la modalité d'assertion: le procès est validé (selon les modalités exprimées par la conjugaison), mais il est privé de la relation syntaxique entre sujet et objet (absence de localisation entre le sujet-agent et objet-patient). L'indétermination spatiale entraîne ici une réfléxivité agentive, un "bouclage" réflexif du procès sur la situation créée par le premier actant, c'est-à-dire l'agent-sujet. L'a-localisation du verbe produit donc une valeur de passif-réfléchi si la portée de -u est le lexème verbal et une valeur de négation si la portée de -u est la prédication.

Tableau 1: Variation d'échelle et emplois de -u

\begin{tabular}{|c|c|}
\hline \multicolumn{2}{|c|}{-u : indétermination par rapport à l'espace-temps de l'énonciation } \\
\hline Portée & Valeur \\
\hline $\begin{array}{l}\text { sur un argument : } \\
\text { sur le temps : } \\
\text { sur le verbe : - en tant que lexème verbal : } \\
\quad \begin{aligned} \text { - en tant que prédicat : }\end{aligned}\end{array}$ & $\begin{array}{l}\text { - connectif, relatif, interrogatif } \\
\text { - subordonnant éventuel ou hypothétique } \\
\text { - passif-réfléchi (objet non localisé) } \\
\text { - négation (procès non localisé) }\end{array}$ \\
\hline
\end{tabular}

On a donc affaire avec -u à un morphème qui fonctionne à différentes échelles syntaxiques, et, tout en subissant une dilatation de sa portée syntaxique, présente au travers de ses différents emplois une structure sémantique similaire. C'est ce que j'ai appelé un "fonctionnement fractal" (Robert 1997). La dilatation change alors le domaine d'application du sémantisme du terme et on passe de la détermination nominale à la prédication ou à la subordination, de l'espace déictique à l'espace des relations de dépendance syntaxique.

\section{DE LA SEMANTIQUE LEXICALE A LA SEMANTIQUE GRAMMATICALE: LA FORME SCHEMATIQUE}

Dans le cas du suffixe -u, l'unité sémantique du morphème paraissait relativement claire. Qu'en est-il lorsque le morphème transcatégoriel présente des emplois à la fois lexicaux et grammaticaux? Le mécanisme de variation d'échelle syntaxique est le même mais, d'une part, l'apparentement sémantique entre les différents emplois du terme peut paraître moins claire, d'autre part, les emplois lexicaux comportent indéniablement une charge sémantique que l'on ne retrouve pas dans les emplois grammaticaux et dont il faut pouvoir rendre compte. Pour définir l'unité sémantique de ce deuxième type de morphème transcatégoriel, on fera intervenir la notion de "forme schématique". Pour rendre compte de la "surcharge sémantique" du lexique par rapport à la grammaire, on fera ensuite intervenir la notion de "propriétés d'échelles" (cf 3.3). Ces différents éléments (variation d'échelle, forme schématique et propriétés d'échelles) constituent les mécanismes qui définissent le modèle de fonctionnement fractal que je propose (cf 3.). Pour en montrer le fonctionnement, je m'appuierai principalement sur l'exemple du wolof ginnaaw et commencerai par présenter la notion de forme schématique, après avoir établi la variation d'échelle.

\subsection{Sémantique et variation d'échelle (l'exemple du wolof ginnaaw)}


Robert, Stéphane, 1999, "Grammaire fractale et sémantique transcatégorielle: entre syntaxe et lexique ", Langages 136 (décembre), numéro consacré à la "Sémantique lexicale et grammaticale" coordonné par Y. Mathieu, 106-123.

Le wolof possède un marqueur ginnaaw qui présente une polysémie particulièrement intéressante puisqu'elle comporte des emplois qui relèvent de trois catégories grammaticales différentes: comme nom, ginnaaw désigne le dos (13), comme préposition, il signifie derrière, après ou excepté (14), et comme conjonction de subordination, il a le sens et les emplois de puisque $^{8}(15)$ :

(13) sama ginnaaw

mon ginnaaw

mon dos

(14a) Mi ngi dëkk ci ginnaaw jàkka ji 3 sg...Présentatif habiter loc. ginnaaw mosquée la

Il habite derrière la mosquée

(14b) Ginnaaw suba ginnaaw demain après-demain ginnaaw añ

ginnaaw déjeûner

après le déjeuner ginnaaw loolu

ginnaaw dém.anaph.

après cela

(14c) Ginnaaw Moodu, ñépp ñëw nañu

ginnaaw Moodu, tous venir Pft.3pl

Excepté Moodu,tous sont venus

(15) Ginnaaw faral nga ko, maa ngi dem.

Ginnaaw soutenir Pft.2sg le 1sg...Présentatif partir

Puisque tu prends son parti, je m'en vais

Tableau 2: Emplois de ginnaaw

\begin{tabular}{|c|c|c|}
\hline ginnaaw & $\begin{array}{l}\text { nom } \\
\text { préposition } \\
\text { conjonction subordination }\end{array}$ & $\begin{array}{l}\text { - dos (partie du corps) } \\
\text { - derrière, après, excepté } \\
\text { - puisque }\end{array}$ \\
\hline
\end{tabular}

J'ai étudié par ailleurs le détail des valeurs d'emploi et les contraintes spécifiques de chacun d'entre eux pour cerner le sémantisme propre du terme et remonter à une valeur commune unitaire (cf Robert 1990b et 1997). Pour les détails de l'argumentation, on pourra se reporter aux travaux mentionnés. Je me contenterai ici d'en présenter les résultats et de montrer comment on peut à la fois poser une valeur commune et retrouver la diversité des emplois à l'aide du modèle fractal.

Les différents emplois de ginnaaw révèlent un schéma d'orientation commun dont le domaine d'application varie. Je pose ainsi que ginnaaw a pour valeur fondamentale de découper un espace dissymétrique et d'indiquer une orientation (devant/derrière) à partir d'un repère.Ginnaaw réfère alors à l'espace situé derrière le repère:

\footnotetext{
${ }^{8}$ En fonction de subordonnant, ginnaaw n'a apparemment jamais de valeur temporelle. Par ailleurs, comme bien souvent en wolof, on relève une fluctuation dans le vocalisme du terme selon les parlers. Il existe en effet une variante gannaaw. Ginnaaw et gannaaw constituent au départ des variantes régionales. On note cependant, dans le parler de Dakar, une tendance récente (non stabilisée) à employer concurremment les deux formes en les spécialisant: gannaaw en emploi lexical et prépositionnel, ginnaaw en fonction subordonnante.
} 
Robert, Stéphane, 1999, "Grammaire fractale et sémantique transcatégorielle: entre syntaxe et lexique ", Langages 136 (décembre), numéro consacré à la "Sémantique lexicale et grammaticale " coordonné par Y. Mathieu, 106-123.

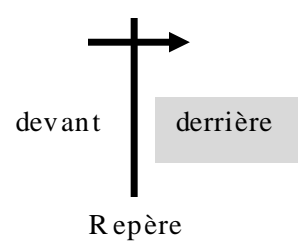

Figure 1: ginnaaw

En faisant varier l'élément qui sert de repère et la nature de l'espace ainsi structuré (domaine d'application), on peut retrouver les différentes valeurs du terme et expliquer leurs contraintes.

(a) En l'absence d'autre terme qui sert de repère, c'est-à-dire en fonction nominale, ginnaaw désigne ce qui se trouve derrière le repère premier c'est-à-dire le corps du sujet: il désigne alors le dos ou plus largement l'espace situé derrière le sujet (16). On notera qu'en tant que nom ginnaaw possède un classificateur ( $\mathrm{g}$-), qu'il peut recevoir les déterminants nominaux, comme le défini (ginnaaw gi: "le dos") ou l'adjectif possessif (13 et 16) ou être introduit par une préposition ci (17):

(16) xoolal ci ginnaawam

regarder-Impér.2sg dans ginnaaw- POSS.3SG

regarde dans son dos / derrière lui

(17) tegal xale bi ci sa ginnaaw

mettre- Impér.2sg enfant DANS ton ginnaaw

mets l'enfant sur ton dos

(b) Lorsqu'un autre nom sert de repère, ginnaaw est en emploi prépositionnel et localise la relation prédicative derrière l'objet qui sert de repère (14a): ci $^{9}$ ginnaaw jàkka ji "derrière la mosquée". Lorsque ginnaaw introduit un terme à valeur temporelle, l'espace ainsi structuré est celui du temps et ginnaaw prend le sens de "après". L'analyse de la valeur d'emploi "excepté" sera présentée plus loin (cf 3.2.) car elle présente d'autres contraintes.

(c) Lorsqu'une proposition $(\mathrm{P})$ sert de repère, ginnaaw indique qu'à partir de cette première proposition, la situation est orientée vers la proposition qui suit $(\mathrm{Q})$ : ginnaaw $\mathrm{P}, \mathrm{Q}$ "puisque $\mathrm{P}$, alors il s'ensuit situation est Q":

(15) Ginnaaw faral nga ko, maa ngi dem.

Puisque tu prends son parti, je m'en vais

glosé: "c'est toi qui l'auras voulu, c'est de ta faute".

Le fait que l'élément repère ne soit plus un nom (comme dans l'emploi prépositionnel) mais une proposition a des conséquences importantes sur la valeur sémantique du terme, nous y reviendrons (cf 2.2. et 3.2.). Disons pour l'instant que l'orientation indiquée par le morphème

\footnotetext{
${ }^{9}$ Ginnaaw, avons-nous dit, réfère à l'espace qui se situe derrière l'élément repère (ici le nom) mais ce nom constitue ici un nouvel argument qui n'a pas de statut syntaxique dans la relation prédicative: on a donc besoin du localisateur $c i$. Par contre, dans le cas de la valeur "après", ginnaaw structure un espace temporel déjà présent au travers de la composante temporelle de la conjugaison (il n'introduit d'ailleurs que des termes qui par euxmêmes impliquent une réfèrence au temps: "le déjeûner", "cela = cet événement"...). Il n'y a donc pas besoin du localisateur. De même dans le cas de "excepté", ginnaaw structure sous forme d'espace le paradigme des sujets (ou des objets) du verbe: il introduit donc également directement le nom.
} 
Robert, Stéphane, 1999, "Grammaire fractale et sémantique transcatégorielle: entre syntaxe et lexique ", Langages 136 (décembre), numéro consacré à la "Sémantique lexicale et grammaticale" coordonné par Y. Mathieu, 106-123.

n'est plus à proprement parler spatiale, mais opère sur des propositions dont elle structure les rapports en termes spatiaux. Cette "spatialisation du discours" définit alors une orientation argumentative (cf 3.2.) que l'on peut paraphraser de la manière suivante: "à partir du fait que [tu prends son parti], il s'ensuit derrière que [je m'en vais]".

\subsection{Forme schématique et échelle syntaxique}

L'invariant sémantique que l'on peut ainsi définir au travers des divers emplois de ginnaaw est une forme abstraite qui n'apparaît jamais directement: elle est toujours instanciée dans un domaine qui varie selon l'élément auquel elle s'applique (le repère de l'orientation). Ce domaine d'application, spécifié par la portée syntaxique de ginnaaw, va produire la polysémie attestée dans les différents emplois du terme. Ainsi, à l'échelle nominale, le domaine d'application de ginnaaw est le corps, à l'échelle prépositionnelle, c'est l'espace (physique) ou temporel et le domaine de la localisation, et à l'échelle propositionnelle, il s'agit des relations entre propositions et de leur relation d'antécédence, causale et discursive. Ce qui fonde ici l'unité sémantique du terme est une configuration très abstraite qui correspond à une forme que l'on retrouve dans tous les emplois, ce que Langacker a appelé 'image schema' (Langacker, 1991), Michaelis (1996) une 'super-structure sémantique' et Culioli une 'forme schématique' (Culioli 1978 et 1987 repris dans Culioli 1990: 115-135). Le terme de 'forme' est ici à entendre au sens gestaltiste de configuration topologique construite par abstraction et qui peut s'appliquer à différents domaines qu'elle va informer. L'intérêt de la notion de forme schématique c'est qu'elle se définit comme une forme génératrice d'autres formes ${ }^{10}$, c'est une sorte de matrice pour produire du sens. Suivant l'échelle syntaxique à laquelle opère le terme, le domaine d'application de cette forme schématique varie, si bien que la forme schématique est à la source de nouvelles formes, par dilatation de sa portée.

\begin{tabular}{|c|c|c|c|}
\hline \multicolumn{4}{|c|}{ Invariant sémantique (forme schématique): } \\
\hline Ginnaaw & \multicolumn{3}{|c|}{$\begin{array}{l}\text { indique une orientation (devant/derrière) à partir d'un repère } \\
\text { et réfère à l'espace situé derrière ce repère }\end{array}$} \\
\hline \multirow[t]{3}{*}{ Variable: } & l'élément qui sert de & epère & \\
\hline & Repère & fonction de ginnaaw & sens \\
\hline & $\begin{array}{l}\text { (1) } \emptyset=\text { le corps } \\
\text { (2) un nom } \\
\text { (3) une proposition }\end{array}$ & $\begin{array}{l}\text { nom } \\
\text { préposition } \\
\text { conj. subord. }\end{array}$ & $\begin{array}{l}\text { dos } \\
\text { derrière, après, excepté } \\
\text { puisque }\end{array}$ \\
\hline
\end{tabular}

A chaque emploi, l'élément qui est en fonction de repère varie et, avec lui, la portée de ginnaaw. D'un emploi à un autre, on a donc préservation (ou abstraction) d'une forme schématique et en même temps activation de propriétés spécifiques. Je vais essayer maintenant de définir ce que sont les propriétés spécifiques de chaque échelle syntaxique et ce qui en déclenche l'activation.

\footnotetext{
${ }^{10}$ La forme schématique "fournit une configuration abstraite qui, selon les transformations qu'on lui fait subir (translation, décrochage, plongement dans un domaine centré, dans un champ de forces intersujets, etc.), va modifier sa forme (marqueur), sa valeur, sa latitude de co-occurrence" (Culioli, 1990: 130).
} 
Robert, Stéphane, 1999, "Grammaire fractale et sémantique transcatégorielle: entre syntaxe et lexique", Langages 136 (décembre), numéro consacré à la "Sémantique lexicale et grammaticale" coordonné par Y. Mathieu, 106-123.

\section{GRAMMAIRE FRACTALE}

Nous avons vu sur ces deux exemples que les morphèmes transcatégoriels présentaient une malléabilité sémantique et syntaxique traversée par des mécanismes de régulation, ce que je propose de décrire comme un "fonctionnement fractal" (cf Robert, 1997b).

\subsection{Fractals et échelles syntaxiques}

En effet, les objets fractals (Mandelbrot 1975), comme une côte maritime, un réseau fluviatile ou une branche d'arbre, ont pour particularité de présenter une structure similaire à différentes échelles: lorqu'on les soumet à une dilatation d'échelle, on observe ainsi à la fois une "invariance d'échelle" (on retrouve une structure analogique) et des "lois d'échelle" ou "propriétés d'échelle" qui font que l'on n'a pas une structure strictement identique d'une échelle à l'autre (Sapoval 1997: 73, 136).

De la même manière, un morphème transcatégoriel présente, au travers de ses différents emplois, une structure sémantique similaire à différentes "échelles", ici syntaxiques: pour expliquer l'unité du terme et l'invariance sémantique, on peut poser que d'un emploi nominal à un emploi prépositionnel puis subordonnant, la structure sémantique du terme reste similaire tout en changeant d'échelle et subissant une "dilatation" de sa portée. En passant d'une échelle à l'autre, le morphème est alors soumis à des "propriétés d'échelles" qui expliquent la spécificité des emplois les uns par rapport aux autres. Ainsi, la nature du domaine d'application du terme varie selon sa portée syntaxique. Or les propriétés structurelles des langues sont telles qu'à chaque fonction syntaxique sont également attachées des propriétés spécifiques. J'illustrerai ces propriétés d'échelles en reprenant plus particulièrement le cas de ginnaaw.

\subsection{Invariance d'échelle: forme schématique}

On a vu que d'un emploi à un autre, il y avait, pour ces morphèmes transcatégoriels, préservation (ou abstraction) d'une forme schématique invariante, qui traversait les différents emplois. Cette forme schématique correspond à l'invariance d'échelle, à la structure analogique qui traverse les échelles et fonde l'unité sémantique du terme. Pour le suffixe -u, cette forme schématique correspond à une indétermination par rapport à l'espace-temps de l'énonciation. Dans le cas de ginnaaw, la forme schématique correspond à une orientation (devant / derrière) à partir d'un repère, telle qu'elle a été représentée dans la figure 1; ginnaaw réfère à l'espace situé derrière ce repère. On peut en effet appliquer ce même schéma d'orientation à tous les emplois du terme, comme nom (cf figure 2), comme préposition (cf figure 3) mais aussi comme subordonnant (cf figure 4). C'est alors seulement la nature de l'élement (le repère) auquel s'applique la forme et donc son domaine d'application qui varie, mais la configuration présente toujours cette même relation d'orientation et de localisation:

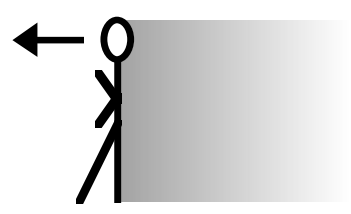

Figure 2: ginnaaw $=$ le DOS 
Robert, Stéphane, 1999, "Grammaire fractale et sémantique transcatégorielle: entre syntaxe et lexique ", Langages 136 (décembre), numéro consacré à la "Sémantique lexicale et grammaticale" coordonné par Y. Mathieu, 106-123.

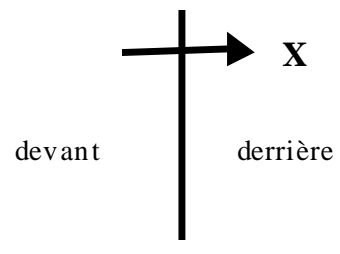

Mosquée
Moodu

Figure 3: Moodu habite DERRIERE la mosquée

Dans le cas de la valeur subordonnante, les éléments en jeu sont des propositions: ginnaaw indique que, selon le point de vue de l'énonciateur, à partir d'une première proposition $\mathrm{P}$ qui sert de repère, l'énoncé est orienté vers ce qui suit, à savoir la proposition Q:

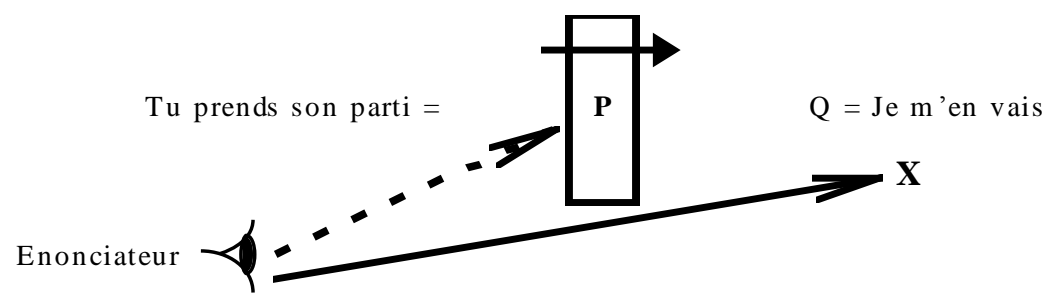

Figure 4: "PUISQUE tu prends son parti, je m'en vais"

Comment arrive-t-on à la valeur "puisque" avec le schéma d'orientation indiqué par ginnaaw? Tout d'abord, la portée de ginnaaw est ici un énoncé complexe et le domaine sur lequel porte l'orientation exprimé par ginnaaw n'est pas celui du temps. En effet, à partir de la "forme" donnée par les relations spatiales, la langue peut choisir de structurer divers domaines. Le domaine d'application est ici celui des relations d'orientation et d'ordonnancement discursif entre propositions (on part d'une proposition et une autre suit derrrière), autrement dit, c'est l'espace discursif que structure la forme schématique de ginnaaw.

On notera de plus que ginnaaw est en tête d'énoncé, sa portée est donc sur l'ensemble de la relation entre les deux propositions, autrement dit, sur l'assertion des liens entre les deux propositions. D'après l'analyse sémantique proposée, la première proposition $\mathrm{P}$ ("tu prends son parti") est le repère derrière lequel la proposition $Q$ ("je m'en vais") est validée. La principale ("je m'en vais") est donc l'objet de l'assertion, le focus de l'énoncé, et la proposition en ginnaaw est présentée comme le repère discursif, le point de départ de l'énoncé: elle a le statut de thème. Le statut thématique de la proposition en ginnaaw est confirmé par les contraintes syntaxiques sur l'ordre des propositions. Contrairement à ce qui se passe pour un autre subordonnant à valeur causale (ndax), la proposition en ginnaaw doit toujours apparaître en premier. L'opposition entre ndax et ginnaaw est comparable à celle qui existe en français entre "je pars parce que tu ne veux pas venir" et "puisque tu ne veux pas venir, je m'en vais". Le statut thématique de la proposition en ginnaaw est confirmé également par l'impossiblité de répondre par ginnaaw à une question en "pourquoi?": la réponse apparaîtrait alors incomplète, il lui manquerait l'information principale (cf "pourquoi tu pars?" "*puisque tu t'en vas").

Ainsi, avec ginnaaw, la protase ("tu prends son parti") est présentée comme le point de départ, donné, à partir duquel, il s'en suit ce qui est validé, à savoir "je m'en vais". On a donc une relation causale entre les deux propositions mais il s'agit d'une causalité particulière, ce que 
Robert, Stéphane, 1999, "Grammaire fractale et sémantique transcatégorielle: entre syntaxe et lexique ", Langages 136 (décembre), numéro consacré à la "Sémantique lexicale et grammaticale " coordonné par Y. Mathieu, 106-123.

j'ai appelé la "causalité argumentative" (Robert 1997b: 122): la première proposition est le point de départ de l'assertion (elle est thématisée) à partir duquel s'ensuit, dans l'espace du discours, la seconde proposition dont elle est donc la source argumentative. Les effets argumentatifs de ginnaaw (comme ceux de puisque en français) découlent de ce statut de repère discursif de la première proposition. Ce point de départ de l'énoncé, l'énonciateur n'en est pas responsable, c'est un fait établi au préalable et indépendant de sa volonté (ou du moins qu'il présente comme tel), à partir duquel lui ne fait que décrire ce qui s'ensuit, la configuration de la situation qui en découle. D'où les gloses relevées: "je n'y peux rien, c'est de ta faute, ce n'est pas moi qui l'ai voulu" et ce que j'ai appelé l'effet "retour à l'envoyeur". Pour plus de détails sur ce point, on pourra se reporter à Robert (1990a) et (1997).

Pour résumer, ce qui est spécifique de l'échelle du discours, c'est que l'élément déterminé par ginnaaw est une proposition qui est thématisée (repère d'énoncé) et qui définit l'orientation du discours (argumentation). Ainsi, l'application à l'échelle des relations interpropositionnelles de la forme schématique définie par ginnaaw produit des effets spécifiques de ce niveau syntaxique. L'organisation spatiale du discours suppose (1) un point de vue: qui définit un "devant" et un "derrière"; ce point de vue est celui de l'énonciateur, instance de prise en charge qui définit la valeur modale de l'énoncé. De plus, (2) l'ordonnancement marqué par ce morphème définit une orientation argumentative à l'intérieur de l'énoncé: à partir d'une première proposition, s'ensuit une autre. Enfin, (3) le repère sur lequel porte ginnaaw a le statut de repère d'un énoncé, il s'agit donc d'un thème (avec les conséquences que l'on vient d'évoquer, sur le statut épistémique de la proposition thématisée) et l'élément que valide ginnaaw est une deuxième proposition qui fonctionne alors comme rhème.

L'emploi de ginnaaw avec le sens de "excepté" confirme cette analyse de l'assertion en termes d'espace assertif.

(14c) Ginnaaw Moodu, ñépp ñëw nañu

Excepté Moodu,tous sont venus

Ici, comme dans de cas de la valeur "puisque" (et contrairement à ce qui se passait pour l'autre emploi prépositionnel "derrière"), ginnaaw doit figurer en tête d'énoncé: sa portée est donc sur l'ensemble de l'assertion. Le morphème spatial introduit un complément mais le syntagme prépositionnel détermine l'ensemble de la proposition. Selon l'analyse générale proposée, ginnaaw crée un espace dissymétrique et valide ce qui est situé derrière le repère (celui-ci n'étant pas inclus). Ainsi, la proposition "ils sont tous venus" est vraie seulement audelà du repère Moodu. D'où le sens "excepté Moodu, tous sont venus".

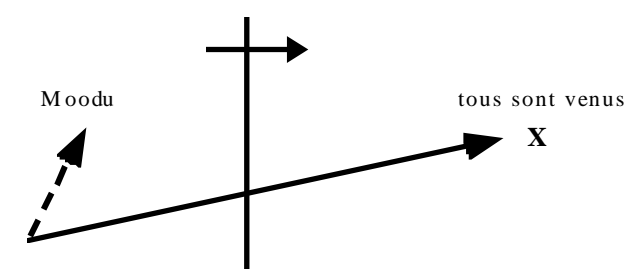

Figure 5 "EXCEPTE Moodu, tous sont venus"

On peut maintenant, revenir à la question de la nature du sémantisme grammatical, tel qu'on a pu le saisir à travers le fonctionnement de ces morphèmes transcatégoriels. Notre modèle fractal confirme tout en la dépassant l'analyse de Talmy (1985) selon laquelle la signification 
Robert, Stéphane, 1999, "Grammaire fractale et sémantique transcatégorielle: entre syntaxe et lexique ", Langages 136 (décembre), numéro consacré à la "Sémantique lexicale et grammaticale" coordonné par Y. Mathieu, 106-123.

grammaticale est fondamentalement topologique et schématique alors que la signification lexicale ne l'est pas. En effet, d'une part, la forme schématique se retrouve dans les emplois lexicaux des termes transcatégoriels, d'autre part, elle permet essentiellement de décrire les liens qui unissent les emplois lexicaux et les emplois grammaticaux d'un même terme, ce qui permet le passage d'un emploi à un autre, autrement dit, le mécanisme invariant qui traverse les échelles. Cependant, comme nous l'avons vu, interviennent également dans la valeur grammaticale de ces morphèmes (tout comme dans chacun de leurs emplois), d'autres propriétés qui produisent des valeurs de sens très spécifiques puisque, dans le cas de ginnaaw, on relève des valeurs argumentatives très fortes dans son emploi le plus grammaticalisé, c'està-dire comme subordonnant. La forme schématique "informe" un domaine au sens où elle lui donne une forme, mais ce domaine a des "propriétés d'échelles" qui déclenchent des facteurs de variation du sens et activent des propriétés sémantiques spécifiques de chaque niveau syntaxique, donc de chacun des emplois. De sorte que l'on ne peut pas se contenter de décrire la sémantique grammaticale comme une simple schématisation. Le passage du lexical au grammatical repose sur une schématisation, mais la sémantique grammaticale ne s'y réduit $\operatorname{pas}^{11}$.

\subsection{Propriétés d'échelles}

Ainsi, le fonctionnement fractal des morphèmes transcatégoriels se caractérise par le fait que (1) on a une forme schématique commune à tous les emplois et qui représente l'invariance d'échelle du morphème, que (2) la portée syntaxique du terme varie dans chacun de ses emplois et déclenche (3) des propriétés d'échelles, spécifiques de chaque emploi et qui procèdent des structures mêmes du langage. Nous avons entrevu ces propriétés d'échelle au cours de l'analyse, j'en donnerai maintenant un bref aperçu général.

Parmi les propriétés d'échelles, figurent, dans tous les cas, des oppositions paradigmatiques variables qui contribuent à la spécification du sens particulier du terme. Nous n'avons guère eu le temps d'y insister mais on retrouve là un phénomène connu. Ainsi, en fonction nominale, ginnaaw rentre dans le paradigme des parties du corps, tel qu'il est construit en wolof. En fonction prépositionnelle, il rentre dans un paradigme de prépositions, qui est d'ailleurs en grande partie formée à partir des parties du corps. En revanche, en fonction de subordonnant, il rentre dans un autre paradigme dont nous avons mentionné l'un des termes, un autre morphème à valeur causale ndax. Ces oppositions paradigmatiques interviennent dans la construction du sens particulier de chaque valeur d'emploi des morphèmes transcatégoriels.

D'autres propriétés d'échelles sont encore plus cruciales pour la sémantique du terme. A l'échelle du nom (et plus généralement du lexique), le morphème a une valeur notionnelle et une portée dénotative. Ainsi, dans les emplois nominaux de ginnaaw, le repère est un élément externe à la relation prédicative et le domaine d'application du terme n'est pas déductible des relations internes à l'énoncé; il s'agit en l'occurrence du corps. Cette valeur notionnelle ne se réduit pas à la forme schématique: elle s'accompagne de diverses connotations, variables selon les langues, d'univers référentiels associés au terme, de propriétés fonctionnelles liées à l'expérience, bref de toute une dimension qui ne se réduit ni au paradigme, ni à la valeur syntagmatique du terme et que j'ai appelée "l'épaisseur du langage" (cf Robert 1997a). Cette fonction dénotative ne se retrouve pas à l'échelle prépositionnelle où apparaît une autre

\footnotetext{
${ }^{11}$ Sur ce point nous rejoignons la position de E. Sweetser (1988: 392-3): la grammaticalisation suppose un transfert de propriétés schématiques et abstraites (et donc une réduction), d'un domaine à un autre, mais dans le transfert, le terme acquiert la signification du nouveau domaine.
} 
Robert, Stéphane, 1999, "Grammaire fractale et sémantique transcatégorielle: entre syntaxe et lexique ", Langages 136 (décembre), numéro consacré à la "Sémantique lexicale et grammaticale " coordonné par Y. Mathieu, 106-123.

propriété d'échelle: celle de relier deux arguments ou un argument et une relation prédicative. Le terme a alors une valeur relationnelle dont le sémantisme est informé par la forme schématique, par le domaine d'application de la préposition et par les propriétés du paradigme des prépositions. A l'échelle de l'énoncé, apparaît un plus grand nombre encore de propriétés d'échelle. Il y a présence d'un point de vue, celui de l'énonciateur, qui va définir la valeur modale de l'énoncé. On a également construction d'un repère discursif (le thème) et d'un focus de l'assertion. Dans le cas d'un énoncé complexe, on a en outre construction de relations interpropositionnelles, et, le cas échéant, d'une orientation argumentative.

\section{Conclusion}

L'étude de ces morphèmes transcatégoriels a permis de voir que la sémantique grammaticale présentait des propriétés communes avec la sémantique lexicale, formulables en termes de formes schématiques de nature topologique. Le modèle fractal proposé permet alors d'expliquer le passage de l'une à l'autre, mais aussi de montrer ce qui les distingue: c'est par des propriétés d'échelles différentes que la sémantique grammaticale se différencie de la sémantique lexicale. En reliant la variation sémantique au changement de portée syntaxique du terme et aux propriétés d'échelle qu'active celui-ci, la grammaire fractale permet ainsi de rendre compte à la fois de l'apparentement entre les divers emplois des morphèmes transcatégoriels et de la variation à l'oeuvre dans chacun d'entre eux. D'un point de vue conceptuel et cognitif, ce modèle dynamique de construction du sens révèle un apparentement remarquable entre des domaines très divers, tels que l'espace déictique et l'espace des relations syntaxiques ou encore le corps, la causalité, l'espace du discours et l'orientation argumentative.

Il convient de préciser que si dans le cas de morphèmes transcatégoriels, la variation est de nature fractale, tous les phénomènes de polysémie ou de grammaticalisation ne sont pas nécessairement de type fractal, ni non plus réductibles à une forme schématique commune. Il existe d'autres configurations par lesquelles les différentes valeurs d'emploi d'un terme sont reliées entre elles. On peut ainsi observer des cas de passage du lexique à la grammaire soit par métonymie, soit par grammaticalisation d'inférences pragmatiques (cf notamment Traugott et Hopper 1993). Dans ce cas, la relation entre les emplois ne passe pas par un invariant commun qui domine l'ensemble des emplois (relation que l'on pourrait dire verticale) mais par un glissement à partir d'une propriété de l'une des valeurs des emplois (relation de type horizontal).

Cependant dans ces différentes configurations, la valeur grammaticale d'un terme entretient toujours avec sa valeur lexicale un rapport d'abstraction par lequel certaines propriétés sont sélectionnées dans le sémantisme lexical pour produire la valeur grammaticale ${ }^{12}$. Ce point est important car il explique d'abord la difficulté que l'on rencontre pour représenter le sémantisme des termes à valeur grammaticale: celui-ci repose sur des propriétés extrêmement abstraites et un apparentement conceptuel entre des domaines parfois très éloignés. Cette sélection de propriétés abstraites qui est à l'oeuvre dans les phénomènes de grammaticalisation

\footnotetext{
12 Je ne prends pas ici position sur la question de l'antériorité d'une valeur sur une autre, du lexical ou concret sur le grammatical ou abstrait Pour mon propos, ce qui importe ici, c'est plus la nature de la relation que son histoire. De ce point de vue, je pense que, s'il existe peut-être des tendances générales ou des cas prototypiques, cependant, les langues présentent différents cas.
} 
Robert, Stéphane, 1999, "Grammaire fractale et sémantique transcatégorielle: entre syntaxe et lexique ", Langages 136 (décembre), numéro consacré à la "Sémantique lexicale et grammaticale " coordonné par Y. Mathieu, 106-123.

explique également un deuxième phénomène. Les domaines sémantiques qui sont à la source des valeurs grammaticales peuvent être très différents puisque, dans le passage à une valeur grammaticale, la langue ne retient qu'un certain nombre de propriétés du sémantisme lexical: ces mêmes propriétés peuvent être extraites de domaines variés. C'est pourquoi les phénomènes de grammaticalisation ou de glissement de sens sont à la fois non aléatoires et non strictement prévisibles. Ainsi, par exemple, l'orientation d'un énoncé vers la suite du discours peut être construite à partir de l'orientation spatiale exprimée par une partie du corps (wolof "dos") ou par une autre (anglais "on the one hand..., on the other hand"), mais aussi à partir de n'importe quel terme qui présente une orientation, dans quelque domaine que ce soit (par exemple, le temps en français avec puis-que). C'est pourquoi la construction du sens par grammaticalisation est à la fois motivée et non aléatoire, mais aussi son strictement déterministe.

\section{Bibliographie}

Culioli, Antoine. 1990. Pour une linguistique de l'énonciation. Opérations et représentations, vol. 1. Paris: Ophrys.

Givón, Talmy. 1975. "Serial verbs and syntactic change: Niger-Congo". In Charles N. Li (ed), Word order and word order change. Austin: University of Texas Press, 17-112.

Hagège, Claude. 1975. Le problème linguistique des prépositions et la solution chinoise (avec un essai de typologie à travers plusieurs groupes de langues), Paris, SLP, Peteers.

Hilaire, Jean-Charles, 1995, Analyse interdialectale de la détermination verbale en peul (parlers centraux du Nigéria), thèse de doctorat, Paris, INALCO.

Langacker, R. W. 1991. "Cognitive Grammar". In F. G. Droste \& J. E. Joseph (eds), Linguistic theory and Grammatical Description, Current issues in linguistic theory 75, Amsterdam / Philadelphia : John Benjamins, 275-306.

Mandelbrot, Benoît. 1975. Les Objets fractals, forme, hasard et dimension. Paris: Flammarion.

Michaelis, Laura A.. 1996, "Cross-world Continuity,and the Polysmey of Adverbial Still". In Gilles Fauconnier and Eve Sweetser (eds), Space, Worlds and Grammar, Chicago and London: The University of Chicago Press, 179-226.

Meillet, Antoine. 1912. "L'Evolution des formes grammaticales". Scientia XII: XXVI, 6. Réimprimé (1948) dans Linguistique Historique et Linguistique Générale 1, Paris: Librairie Honoré Champion, 130-48.

Robert, Stéphane. 1990a. "Puisque et le dos en wolof, tempête dans le signifié". Le Gré des langues 1: 82-92.

Robert, Stéphane. 1990b, “Aperçu sur la négation en wolof”, Linguistique africaine $\mathrm{n}^{\circ} 4$, Documents de travail sur la négation, Paris, 167-180.

Robert, Stéphane. 1991, Une approche énonciative du système verbal : le cas du wolof. Paris: Editions du CNRS (collection Sciences du langage).

Robert, Stéphane. 1996. Aspect zéro et dépendance situationnelle : l'exemple du wolof. In Claude Muller (ed.), Dépendance et intégration syntaxique. Tübingen: Niemeyer, 153-161.

Robert, Stéphane, 1997a, "Variations des représentations linguistiques : des unités à l'énoncés". In Catherine Fuchs et Stéphane Robert (éds), Diversité des langues et représentations cognitives, Paris/Gap: Ophrys, 25-39.

Robert, Stéphane. 1997b, "From body to argumentation: grammaticalization as a fractal property of language (the case of Wolof ginnaaw)", Berkeley Linguistics Society 23S, 
Robert, Stéphane, 1999, "Grammaire fractale et sémantique transcatégorielle: entre syntaxe et lexique", Langages 136 (décembre), numéro consacré à la "Sémantique lexicale et grammaticale" coordonné par Y. Mathieu, 106-123.

Proceedings of the 23rd Annual Meeting (14-16 février 1997), Special Session on Syntax and Semantics in African Languages, Berkeley, 116-127.

Robert, Stéphane. 1998, "Espace déictique, espace syntaxique et prédication : les indices spatiaux du wolof'. In B. Caron (ed), Proceedings of the 16th International Congress of Linguists (CD Rom), CNRS-LLACAN Meudon / Amsterdam: Elsevier.

Sapoval, Bernard. 1997. Universalité et fractales. Paris: Flammarion.

Sauvageot, Serge. 1965, Description synchronique d'un dialecte wolof, le parler du Dyolof, Dakar: IFAN.

Sweetser, Eve, 1988. "Grammaticalization and semantic bleaching". Berkeley Linguistics Society 14, Proceedings of the 14th Annual Meeting of the Berkeley Linguistics Society. Berkeley: Berkeley Linguistics Society, 389-409.

Talmy, Leonard. 1985, "Lexicalization pattern: semantic structure in lexical forms". In Timothy Shopen (ed), Language Typology and Syntactic Description (Vol.3: Grammatical Categories and the Lexicon). Cambridge: Cambridge University Press, 57-148.

Traugott, Elisabeth Closs and Paul Hopper, 1993, Grammaticalization. Cambridge: Cambridge University Press. 